УДК $517.55+519.1$

\title{
Method for Obtaining Combinatorial Identities with Polynomial Coefficients by Means of Integral Representations
}

\author{
Viacheslav P. Krivokolesko* \\ Institute of Mathematics and Computer Science \\ Siberian Federal University \\ Svobodny, 79, Krasnoyarsk, 660041
}

Russia

Received 06.12.2015, received in revised form 08.01.2016, accepted 12.02.2016

We propose a method how to derive combinatorial identities with polynomial coefficients by means of an integral representation of holomorphic functions in an n-circular linearly convex polyhedron.

Keywords: combinatorial identities, polynomial coefficients, integral representation.

DOI: 10.17516/1997-1397-2016-9-2-192-201.

\section{Introduction}

In [1] a number of some combinatorial identities was obtained. One of such identities is a generalization of the Chaundy-Bullard identity [2], see also [3-10]

The proof was based on integration of holomorphic monomials along a piecewise regular boundary of a bounded linearly convex $n$-circular domain in $\mathbb{C}^{n}$, in particular, of a bicircular domain in $\mathbb{C}^{2}$.

Similar identities were obtained in [11], where holomorphic monomials were integrated along the boundary of a 3 -circular domain in $\mathbb{C}^{3}$.

These identities were verified and generalized by the method of Egorychev from [12] developed in [13]

The domains considered in [1] and [11] were not complete and the identities obtained have binomial and polynomial coefficients.

The structure of the integral representation [14] and known examples imply the following conjecture

Problem 1. The integral representation in [14] permits one to obtain combinatorial identities related to the geometry of a domain, along whose boundary the integration of a holomorphic function is performed.

As follows from the proof of theorem 6 of this paper, this conjecture is proved to be true for complete bounded linearly convex domains with piecewise regular boundary in $\mathbb{C}^{n}$.

The article consists of three sections, the first two preceed the proof of the main theorem.

In the first section we formulate the theorem on the integral representation in bounded n-circular linearly convex domains with piecewise regular boundary in $\mathbb{C}^{n}$ and introduce the necessary notation and definitions.

In the second section we study some properties of the projection $\pi: \mathbb{C}^{n} \rightarrow R_{+}^{n}$, where $R_{+}^{n}$ is the nonnegative part of $R^{n}$ (i.e the Reihardt diagram centered at the origin).

In the last section we formulate and prove a lemma and the main theorem.

*krivokolesko@gmail.com

(c) Siberian Federal University. All rights reserved 


\section{Integral representation in n-circular linearly convex domains with piecewise regular boundary in $\mathbb{C}^{n}$}

A domain $G \subset \mathbb{C}^{n}$ is called linearly convex $([15], \S 8)$, if for each point $z_{0}$ of its boundary $\partial G$ there is a complex $(n-1)$-dimensional analytic plane passing through the point $z_{0}$ and disjoint from $G$ (note that some authors use the term "weak linear convexity", for example, see [16]).

Let there be given a bounded linearly convex domain of the polyhedral form (a linearly convex polyhedron)

$$
G=\left\{z: g^{l}(z, \bar{z})<0, \quad l=1, \ldots, N\right\}
$$

in the space $\mathbb{C}^{n}$, where the functions $g^{l}(z, \bar{z})$ are twice continuously differentiable in a neighborhood of the closure of the domain.

The boundary $\partial G$ of the domain $G$ is called piecewise regular, if for every nonempty edge

$$
\begin{gathered}
S^{J_{k}}=S^{j_{1} \ldots j_{k}}=S^{j_{1}} \cap \ldots \cap S^{j_{k}}= \\
=\left\{\zeta \in \partial G: g^{j_{1}}(\zeta, \bar{\zeta})=0, \ldots, g^{j_{k}}(\zeta, \bar{\zeta})=0,1 \leqslant k \leqslant n\right\}
\end{gathered}
$$

we have the inequality $\bar{\partial} g^{j_{1}} \wedge \ldots \wedge \bar{\partial} g^{j_{k}} \neq 0$, or equivalently

$$
\operatorname{rang}\left(\begin{array}{ccc}
\frac{\partial g^{j_{1}}}{\partial \bar{\zeta}_{1}} & \ldots & \frac{\partial g^{j_{1}}}{\partial \bar{\zeta}_{n}} \\
\ldots & \ddots & \ldots \\
\frac{\partial g^{j_{k}}}{\partial \bar{\zeta}_{1}} & \cdots & \frac{\partial g^{j_{k}}}{\partial \bar{\zeta}_{n}}
\end{array}\right)=k .
$$

The orientation of the faces $S^{1}, \ldots, S^{N}$ is induced by the orientation of the boundary $\partial G, \partial G=$ $\bigcup_{i=1}^{N} S^{i}$ and the orientation of each face $S^{i}, i=1, \ldots, N$ induce the orientation of every $(2 n-2)$ dimensional edge $S^{i j}, \partial S^{i}=\bigcup_{j} S^{i j}$, and, taking into account the orientation, we have $S^{i j}=-S^{j i}$. The orientation of the edge $S^{j_{1} \ldots j_{k}}$ is given by ordering of the faces $S^{j_{1}}, \ldots, S^{j_{k}}$, and is defined inductively.

The integral representation in bounded linearly convex domains with piecewise boundary is proved in [14]. In [1] we give a detalization of the key theorem from [14] for $n$-circular domains, using which we derive a series of combinatorial identities.

Next we need the formulation of the theorem from [1].

\subsection{Detalization of the integal representation from [14]}

A domain $G \subset \mathbb{C}^{n}$ is $n$-circular if for each point $z=\left(z_{1}, \ldots, z_{n}\right) \in G$ it contains all points $z \cdot e^{i \varphi}=\left(z_{1} \cdot e^{i \varphi_{1}}, \ldots, z_{n} \cdot e^{i \varphi_{n}}\right), 0 \leqslant \varphi_{1}<2 \pi, \ldots, 0 \leqslant \varphi_{n}<2 \pi$.

In case of an $n$-circular polyhedron the functions $g^{l}$ depend on modules $\left|z_{j}\right|$ only, i. e. such polyhedra are as follows

$$
G=\left\{z: g^{l}(|z|)<0, \quad l=1, \ldots, N\right\}
$$

where the functions $g^{l}(|z|)$ are twice continuously differentiable in a neighborhood of the closure of this domain.

Introduce the following notation:

$$
g_{|m|}^{l}=\frac{\partial g^{l}}{\partial\left|\zeta_{m}\right|}, \quad g_{|s||m|}^{l}=\frac{\partial^{2} g^{l}}{\partial\left|\zeta_{s}\right| \partial\left|\zeta_{m}\right|}
$$




$$
\begin{aligned}
& \left\langle\nabla g^{l},\right\rangle=\left\langle\nabla g_{|\zeta|}^{l},|\zeta|-\xi z\right\rangle=\sum_{m=1}^{n} g_{|m|}^{l}\left(\left|\zeta_{m}\right|-\xi_{m} z_{m}\right), \\
& \tilde{c}_{s m}=\sum_{t=1}^{k} \frac{\lambda_{t}}{\left\langle g^{t},\right\rangle}\left(\left|\zeta_{s}\right| g_{|s||m|}^{t}+\delta_{s}^{m} g_{|m|}^{t}\right), \quad \sum_{t=1}^{k} \lambda_{t}=1, \quad \lambda_{t} \geqslant 0, t=1, \ldots, k \\
& \tilde{R}^{J_{k}}=-\left|\begin{array}{cccccc}
0 & \ldots & 0 & g_{|1|}^{j_{1}} & \ldots & g_{|n|}^{j_{1}} \\
\ldots & \ddots & \ldots & \ldots & \ddots & \ldots \\
0 & \ldots & 0 & g_{|1|}^{j_{k}} & \ldots & g_{|n|}^{j_{k}} \\
\left|\zeta_{1}\right| g_{|1|}^{j_{1}} & \ldots & \left|\zeta_{1}\right| g_{|1|}^{j_{k}} & \tilde{c}_{11} & \ldots & \tilde{c}_{1 n} \\
\ldots & \ddots & \ldots & \ldots & \ddots & \ldots \\
\left|\zeta_{n}\right| g_{|n|}^{j_{1}} & \ldots & \left|\zeta_{n}\right| g_{|n|}^{j_{k}} & \tilde{c}_{n 1} & \ldots & \tilde{c}_{n n}
\end{array}\right|, \\
& \tilde{\Delta}_{j_{1} \ldots j_{k}}^{p_{1} \ldots p_{k}}=\left|\begin{array}{ccc}
g_{\left|p_{1}\right|}^{j_{1}} & \ldots & g_{\left|p_{k}\right|}^{j_{1}} \\
\ldots & \ddots & \ldots \\
g_{\left|p_{1}\right|}^{j_{k}} & \ldots & g_{\left|p_{k}\right|}^{j_{k}}
\end{array}\right| .
\end{aligned}
$$

We denote

$$
\tilde{\omega}_{J_{k}}=\frac{(-1)^{\frac{k(k+1)}{2}} \cdot(-1)^{p_{1}+\ldots+p_{k}} d|\zeta|\left[p_{1}, \ldots, p_{k}\right]}{\tilde{\Delta}_{j_{1} \ldots j_{k}}^{p_{1} \ldots p_{k}}},
$$

where $\tilde{\Delta}_{j_{1} \ldots j_{k}}^{p_{1} \ldots p_{k}} \neq 0$ and $d|\zeta|\left[p_{1}, \ldots, p_{k}\right]$ is the exterior product of the differentials $d\left|\zeta_{1}\right|, \ldots, d\left|\zeta_{n}\right|$, where $d\left|\zeta_{p_{1}}\right|, \ldots, d\left|\zeta_{p_{k}}\right|$ are omitted.

Note that if $1 \leqslant k \leqslant n$ we find the mixed Levians $\tilde{L}_{I}\left(g^{j_{1}}, \ldots, g^{j_{k}}\right)=\tilde{L}_{I_{k}}\left(g^{J_{k}}\right)$ from

$$
\tilde{R}^{J_{k}}=\tilde{R}^{j_{1} \ldots j_{k}}=\sum_{|I|=n-k} \frac{\lambda_{1}^{i_{1}} \ldots \lambda_{k}^{i_{k}}}{\left\langle g_{|\zeta|}^{j_{1}},|\zeta|-\xi z\right\rangle^{i_{1}} \ldots\left\langle g_{|\zeta|}^{j_{k}},|\zeta|-\xi z\right\rangle^{i_{k}}} \cdot \tilde{L}_{I}\left(g^{j_{1}}, \ldots, g^{j_{k}}\right) .
$$

Notation (2)-(8) allow us to formulate the following theorem from [1].

Theorem 2. Let $G=\left\{z: g^{l}(|z|)<0, l=1, \ldots, N\right\}$ be a bounded piecewise regular linearly convex domain in $\mathbb{C}^{n}$. Then every function $f(z)$ holomorphic in $G$ and continious on $\bar{G}$ is representable in $G$ as

$$
f(z)=(-1)^{n} \sum_{k=1}^{n}(-1)^{k-1} \sum_{\sharp J=k} \sum_{|I|=n-k} \nu^{j_{1} \ldots j_{k}},
$$

where $\sum_{\sharp J_{k}=k}^{\prime}$ stands for summation over ordered multi-indices $J_{k}$ of length $k: 1 \leqslant j_{1}<\ldots<$ $j_{k} \leqslant N ; \sum_{\left|I_{k}\right|=n-k}$ stands for summation over multi-indices $I_{k}=\left(i_{1}, \ldots, i_{k}\right)$ with the property $\left|I_{k}\right|:=i_{1}+\ldots+i_{k}=n-k$,

$$
\nu^{j_{1} \ldots j_{k}}=\frac{I !}{(2 \pi i)^{n}} \int_{\left|S^{J}\right|} \tilde{L}_{I}\left(g^{j_{1}}, \ldots, g^{j_{k}}\right) \tilde{\omega}_{J} \int_{|\xi|=1} \frac{f\left(\frac{\left|\zeta_{1}\right|}{\xi_{1}}, \ldots, \frac{\left|\zeta_{n}\right|}{\xi_{n}}\right)}{\prod_{t=1}^{k}\left\langle\nabla g_{|\zeta|}^{j_{t}},|\zeta|-\xi z\right\rangle^{i_{t}+1}} \cdot \frac{d \xi}{\xi},
$$

$\frac{d \xi}{\xi}=\frac{d \xi_{1}}{\xi_{1}} \wedge \ldots \wedge \frac{d \xi_{n}}{\xi_{n}}, \tilde{L}_{I}$ is a mixed Levian, and $I_{k} !=i_{1} ! \cdot \ldots \cdot i_{k} !$ 


\subsection{Some properties of the projection of $\mathbb{C}^{n}$ on $R_{+}^{n}$}

For a point $z=\left(z_{1}, \ldots, z_{n}\right) \in \mathbb{C}^{n}$ we denote $|z|=\left(\left|z_{1}\right|, \ldots,\left|z_{n}\right|\right) \in R_{+}^{n}$.

Consider a projection of $\mathbb{C}^{n}$ on the Reinhardt diagram $\left(R_{+}^{n}\right)$

$$
\pi:\left(z_{1}, \ldots, z_{n}\right) \rightarrow\left(\left|z_{1}\right|, \ldots,\left|z_{n}\right|\right) .
$$

We denote the image of the set $G \subset \mathbb{C}^{n}$ under this projection as $|G| \subset R_{+}^{n}$.

The projection $\pi$ maps $n$-circular sets $G \subset \mathbb{C}^{n}$ bijectively to sets $|G|=\pi(G) \subset R_{+}^{n}$.

Let

$$
a_{1} z_{1}+\ldots+a_{n} z_{n}+c=0
$$

be an analytic plane in the space $\mathbb{C}^{n}$ that passes through the point $\zeta\left(c=-\left(a_{1} \zeta_{1}+\ldots+a_{n} \zeta_{n}\right)\right)$ disjoint from the domain $G \in \mathbb{C}^{n}$.

The description of the projection (12) on the Reinhardt diagram is given in Proposition 4.3 [17].

Projection (12) is given by the system of inequalities:

$$
\left\{\begin{array}{l}
+\left|a_{1}\right|\left|z_{1}\right|-\left|a_{2}\right|\left|z_{2}\right|-\left|a_{3}\right|\left|z_{3}\right|-\ldots-\left|a_{n}\right|\left|z_{n}\right|-|c| \leqslant 0, \\
-\left|a_{1}\right|\left|z_{1}\right|+\left|a_{2}\right|\left|z_{2}\right|-\left|a_{3}\right|\left|z_{3}\right|-\ldots-\left|a_{n}\right|\left|z_{n}\right|-|c| \leqslant 0, \\
\vdots \\
-\left|a_{1}\right|\left|z_{1}\right|-\left|a_{2}\right|\left|z_{2}\right|-\left|a_{3}\right|\left|z_{3}\right|-\ldots-\left|a_{n}\right|\left|z_{n}\right|+|c| \leqslant 0 .
\end{array}\right.
$$

Let us assume that an analytic plane (12) passes through the point $\zeta$ i.e. $c=-\left(a_{1} \zeta_{1}+\ldots+a_{n} \zeta_{n}\right)$. Then each plane of the family

$$
a_{1} z_{1} e^{-i \varphi_{1}}+\ldots+a_{n} z_{n} e^{-i \varphi_{n}}+c=0, \quad 0 \leqslant \varphi_{m}<2 \pi, \quad m=1, \ldots, n
$$

passes respectively through point $\zeta e^{i \varphi}=\left(\zeta e^{i \varphi_{1}}, \ldots, \zeta e^{i \varphi_{n}}\right)$.

It follows that the set (14) is an $n$-circular set in $\mathbb{C}^{n}$. Moreover its projection on the Reinhardt diagram is given by the system (13) and coincides with the projection of each plane on the Reinhardt diagram of the family (14).

The properties of the projection $\pi$ for $n$-circular sets give us the following geometric criterion of linear convexity of a domain $G \subset \mathbb{C}^{n}$ at points $\zeta$ of the boundary $\partial G$.

Criterion 3. Let an analitic plane (12) be disjoint from an $n$-circular domain $G \subset \mathbb{C}^{n}$ and pass through the point $\zeta \in \partial G$ the boundary of the domain $G$. Then the system of inequalities (13) on the Reinhardt diagram is disjoint from the domain $|G|=\pi(G)$, $|\zeta|$ belongs to the closure of the set given by the system (13), and $|\zeta| \in \partial|G|$.

Let the set given by (13) be disjoint from the domain $B \subset R_{+}^{n}$ and $|\zeta| \in \partial B$ belong to the closure of the set given by the system (13), then the $n$-circular family of planes in $\mathbb{C}^{n}$

$$
\left|a_{1}\right| z_{1} e^{-i \varphi_{1}}+\ldots+\left|a_{n}\right| z_{n} e^{-i \varphi_{n}}+c=0, \quad 0 \leqslant \varphi_{m}<2 \pi, \quad m=1, \ldots, n
$$

disjoint from the domain $G=\pi^{-1}(B) \subset \mathbb{C}^{n}$ and passing through the point $|\zeta| e^{i \varphi} \in \partial G$ corresponds to the system (13).

Definition 1. A set $G \subset \mathbb{C}^{n}$ is called complete if together with the point $z^{0}=\left(z_{1}^{0}, \ldots, z_{n}^{0}\right) \in G$ the points $\left\{z=\left(z_{1}, \ldots, z_{n}\right):\left|z_{1}\right| \leqslant\left|z_{1}^{0}\right|, \ldots,\left|z_{n}\right| \leqslant\left|z_{n}^{0}\right|\right\}$ belong to $G$.

It follows from the definition that a complete set $G \subset \mathbb{C}^{n}$ is $n$-circular, since with each point $z=\left(z_{1}, \ldots, z_{n}\right) \in G$ it contains all the points $z \cdot e^{i \varphi}=\left(z_{1} \cdot e^{i \varphi_{1}}, \ldots, z_{n} \cdot e^{i \varphi_{n}}\right), 0 \leqslant \varphi_{1}<$ $2 \pi, \ldots, 0 \leqslant \varphi_{n}<2 \pi$, and also contains the origin. 


\subsubsection{Some properties of $\pi$}

Define in $R_{+}^{n}$ the following "planes " :

$$
\begin{aligned}
& +\left|a_{1}\right|\left|z_{1}\right|-\left|a_{2}\right|\left|z_{2}\right|-\left|a_{3}\right|\left|z_{3}\right|-\ldots-\left|a_{n}\right|\left|z_{n}\right|-|c|=0, \ldots, \quad\left(\Pi_{1}\right) \\
& -\left|a_{1}\right|\left|z_{1}\right|-\left|a_{2}\right|\left|z_{2}\right|-\ldots-\left|a_{n-1}\right|\left|z_{n-1}\right|+\left|a_{n}\right|\left|z_{n}\right|-|c|=0, \quad\left(\Pi_{n}\right) \\
& -\left|a_{1}\right|\left|z_{1}\right|-\left|a_{2}\right|\left|z_{2}\right|-\left|a_{3}\right|\left|z_{3}\right|-\ldots-\left|a_{n}\right|\left|z_{n}\right|+|c|=0, \quad\left(\Pi_{n+1}\right)
\end{aligned}
$$

and "spaces"

$$
\begin{array}{lr}
+\left|a_{1}\right|\left|z_{1}\right|-\left|a_{2}\right|\left|z_{2}\right|-\left|a_{3}\right|\left|z_{3}\right|-\ldots-\left|a_{n}\right|\left|z_{n}\right|-|c|>0, \ldots, & \left(+\Pi_{1}\right) \\
-\left|a_{1}\right|\left|z_{1}\right|-\left|a_{2}\right|\left|z_{2}\right|-\ldots-\left|a_{n-1}\right|\left|z_{n-1}\right|+\left|a_{n}\right|\left|z_{n}\right|-|c|>0, & \left(+\Pi_{n}\right) \\
-\left|a_{1}\right|\left|z_{1}\right|-\left|a_{2}\right|\left|z_{2}\right|-\left|a_{3}\right|\left|z_{3}\right|-\ldots-\left|a_{n}\right|\left|z_{n}\right|+|c|>0 . & \left(+\Pi_{n+1}\right)
\end{array}
$$

It is evident thNauka, at $+\Pi_{l} \cap+\Pi_{m}=\varnothing$ when $l \neq m ; l, m=1, \ldots, n+1$ and $+\Pi_{l} \cap(13)=\varnothing$, when $l=1, \ldots, n+1$ and $+\Pi_{1} \cup \ldots \cup+\Pi_{n+1} \cup(13)=R_{+}^{n}$.

Therefore

(a) if an analytic plane $a_{1} z_{1}+\ldots+a_{n} z_{n}+c=0$ in $\mathbb{C}^{n}$ is disjoint from an $n$-circular domain $G \in \mathbb{C}^{n}$ then $|G|$ belongs to one of the "spaces" $+\Pi_{1}, \ldots,+\Pi_{n+1}$ in $R_{+}^{n}$;

(b) if an analytic plane $a_{1} z_{1}+\ldots+a_{n} z_{n}+c=0$ in $\mathbb{C}^{n}$ is disjoint from an $n$-circular domain $G \in \mathbb{C}^{n}$ and passes through the point $\zeta \in \partial G$ then the point $|\zeta|$ belongs to one of the "planes" $\Pi_{m}$ when $m=1, \ldots, n+1$ which is tangent to $|G|$ at the point $|\zeta| \in \partial|G|$;

Remark 4. If $n$-circular domain $G \subset \mathbb{C}^{n}$ is linearly convex then $|G|$ is convex in $R_{+}^{n}$.

The converse is false, an example is given by an open ball in $R_{+}^{n}$.

(c) if an analytic plane $a_{1} z_{1}+\ldots+a_{n} z_{n}+c=0$ in $\mathbb{C}^{n}$ is disjoint from a complete domain $G \in \mathbb{C}^{n}$ then $|G|$ belongs to $+\Pi_{n+1}$ i.e. for points of a complete $n$-circular domain $G \in \mathbb{C}^{n}$ there is an inequality:

$$
\left|a_{1}\right|\left|z_{1}\right|+\left|a_{2}\right|\left|z_{2}\right|+\ldots+\left|a_{n}\right|\left|z_{n}\right|-|c|<0
$$

from which follows

$$
|\langle a, z\rangle|=\left|a_{1} z_{1}+a_{2} z_{2}+\ldots+a_{n} z_{n}\right|<|c| .
$$

Remark 5. Recall that we consider a bounded linearly convex domain

$$
G=\left\{z: g^{l}(z, \bar{z})<0, \quad l=1, \ldots, N\right\}
$$

where the functions $g^{l}(z, \bar{z})$ are twice continuously differentiable in a neighborhood of the closure of the domain. The boundary $\partial G$ of $G$ consists of the faces

$$
S^{l}=\left\{z \in \bar{G}: g^{l}(z, \bar{z})=0\right\}, \quad l=1, \ldots, N .
$$

The analytic plane tangent to the domain $G$ at the point $\zeta \in S^{l}$ ([15], p. 87), i. e. which passes through the point $\zeta \in \partial G$ and is disjoint from $G$ is given by:

$$
\left\{z:\left\langle\nabla g_{\zeta}^{l}, z-\zeta\right\rangle=\sum_{m=1}^{n} \frac{\partial g^{l}}{\partial \zeta_{m}}\left(z_{m}-\zeta_{m}\right)=0\right\} .
$$

Note that the number of all analytic planes tangent to the domain $G$ at the point $\zeta$ of the edge $S^{j_{1} \ldots j_{k}} \subset \partial G$ is described by analytic planes tangent to the faces $S^{j_{1}}, \ldots, S^{j_{k}}$ at the point $\zeta$. 
From (20) we see that in case of $n$-circular domains analytic planes tangent to the domain $G$ at the point $\zeta e^{i \varphi} \in S^{l}, 0 \leqslant \varphi<2 \pi$ are given by

$$
\left\{z:\left\langle\nabla g_{|\zeta|}^{l},|\zeta|-e^{-i \varphi} z\right\rangle=\sum_{m=1}^{n} \frac{\partial g^{l}}{\partial\left|\zeta_{m}\right|}\left(\left|\zeta_{m}\right|-e^{-i \varphi_{m}} z_{m}\right)=0\right\} .
$$

Applying the notation (2) we write the equations of the family analytic tangent planes (21) as:

$$
\sum_{m=1}^{n} g_{|m|}^{l} \xi_{m} z_{m}-\sum_{m=1}^{n} g_{|m|}^{l}\left|\zeta_{m}\right|=0
$$

where $\left|\xi_{1}\right|=\ldots=\left|\xi_{m}\right|=1$.

For the points $z$ of a complete linearly convex domain $G$ and $\left|\xi_{1}\right|=\ldots=\left|\xi_{m}\right|=1$ by virtue of (19), we have

$$
\left|\sum_{m=1}^{n} g_{|m|}^{l} \xi_{m} z_{m}\right|<\left|\sum_{m=1}^{n} g_{|m|}^{l}\right| \zeta_{m} \mid
$$

It follows from (23) that for points $z$ of a complete linearly convex domain $G$ and $\left|\xi_{1}\right|=\ldots=$ $\left|\xi_{m}\right|=1$

$$
\sum_{m=1}^{n} g_{|m|}^{l}\left|\zeta_{m}\right|-\sum_{m=1}^{n} g_{|m|}^{l} \xi_{m} z_{m}=A_{l}\left(1-\sum_{m=1}^{n} \frac{g_{|m|}^{l} \xi_{m} z_{m}}{A_{l}}\right)=A_{l}\left(1-\left\langle\mathbf{a}_{\mathbf{l}}, \xi\right\rangle\right)
$$

where

$$
\left|\left\langle\mathbf{a}_{\mathbf{l}}, \xi\right\rangle\right|=\left|\sum_{m=1}^{n} a_{l m} \xi_{m}\right|<1
$$

and

$$
A_{l}=\sum_{m=1}^{n} g_{|m|}^{l}\left|\zeta_{m}\right|, \mathbf{a}_{\mathbf{l}}=\left(\frac{g_{|1|}^{l} z_{1}}{A_{l}}, \ldots, \frac{g_{1}^{l} z_{1}}{A_{l}}\right)
$$

\section{The main theorem}

Theorem 6. For every bounded and complete linearly convex domain $G$ with piecewise regular boundary such that all the vertices $S^{j_{1} \ldots j_{n}}$ are distinct there is a combinatorial identity with binomial coefficients

$$
1=(-1)^{n} \sum_{k=1}^{n}(-1)^{k-1} \sum_{\sharp J_{k}=k} \sum_{\left|I_{k}\right|=n-k} \sum_{\mathbf{m}_{\mathbf{J}_{\mathbf{k}}}=s} \frac{\left(\left|\mathbf{m}_{\mathbf{j}_{1}}\right|+i_{1}\right) ! \cdot \ldots \cdot\left(\left|\mathbf{m}_{\mathbf{j}_{\mathbf{k}}}\right|+i_{k}\right) !}{\mathbf{m}_{\mathbf{j}_{\mathbf{1}}} ! \cdot \ldots \cdot \mathbf{m}_{\mathbf{j}_{\mathbf{1}}} !} \Omega_{\mathbf{m}_{\mathbf{J}_{\mathbf{k}}}}\left(s, J_{k}, I_{k}\right),
$$

where

$$
\Omega_{\mathbf{m}_{\mathbf{J}_{\mathbf{k}}}}\left(s, J_{k}, I_{k}\right)=\int_{\mid S^{J_{k} \mid}} \frac{|\zeta|^{s}\left(g_{|\zeta|}^{j_{1}}\right)^{\mathbf{m}_{\mathbf{j}_{1}}} \cdot \ldots \cdot\left(g_{|\zeta|}^{j_{k}}\right)^{\mathbf{m}_{\mathbf{j}_{\mathbf{k}}}}}{\prod_{t=1}^{k}\left(A_{j_{t}}\right)^{\left|\mathbf{m}_{\mathbf{j}_{\mathbf{t}}}\right|+i_{t}+1}} \cdot \tilde{L}_{I_{k}}\left(g^{J_{k}}\right) \cdot \omega_{J_{k}}
$$

and

$$
\begin{gathered}
A_{j_{t}}=\sum_{l=0}^{n} g_{\zeta_{l}}^{j_{t}}\left|\zeta_{l}\right|, \quad g_{|\zeta|}^{j_{t}}=\left(g_{1}^{j_{t}}, \ldots, g_{n}^{j_{t}}\right), \quad|\zeta|^{s}=\left|\zeta_{1}\right|^{s_{1}} \cdot \ldots \cdot\left|\zeta_{n}\right|^{s_{n}} \\
\mathbf{a}_{\mathbf{j}_{\mathbf{t}}}=\left(a_{j_{1} 1}, \ldots, a_{j_{t} n}\right), \mathbf{m}_{\mathbf{j}_{\mathbf{t}}}=\left(m_{j_{1} 1}, \ldots, a_{j_{t} n}\right) \\
-197-
\end{gathered}
$$




$$
\begin{gathered}
\mathbf{a}_{\mathbf{j}_{\mathbf{t}}}^{\mathbf{m}_{\mathbf{j}_{\mathbf{t}}}}=\prod_{l=1}^{n} a_{j_{t} l}^{m_{j_{t} l} l}, \mathbf{m}_{\mathbf{j}_{\mathbf{t}}} !=\prod_{l=1}^{n} m_{j_{t} l} !,\left|\mathbf{m}_{\mathbf{j}_{\mathbf{t}}}\right|=m_{j_{t} 1}+\ldots+m_{j_{t} n}, \\
\mathbf{a}_{\mathbf{J}_{\mathbf{k}}} \mathbf{m}_{\mathbf{k}_{\mathbf{k}}} \\
=\prod_{t=1}^{k} \mathbf{a}_{\mathbf{j}_{\mathbf{t}}}^{\mathbf{m}_{\mathbf{t}}}, \quad \mathbf{m}_{\mathbf{J}_{\mathbf{k}}} !=\prod_{\mathbf{t}=\mathbf{1}}^{\mathbf{k}} \mathbf{m}_{\mathbf{j}_{\mathbf{t}}} !
\end{gathered}
$$

and

$$
\begin{gathered}
\mathbf{m}_{\mathbf{J}_{\mathbf{k}}}=\mathbf{m}_{\mathbf{j}_{1}}+\ldots+\mathbf{m}_{\mathbf{j}_{\mathbf{k}}}=\left(m_{j_{1} 1}+\ldots+m_{j_{k} 1}, \ldots, m_{j_{1} n}+\ldots+m_{j_{k} n}\right) \\
\left|\mathbf{m}_{\mathbf{J}_{\mathbf{k}}}\right|=m_{j_{1} 1}+\ldots+m_{j_{k} 1}+\ldots+m_{j_{1} n}+\ldots+m_{j_{k} n}=\left|\mathbf{m}_{\mathbf{j}_{1}}\right|+\ldots+\left|\mathbf{m}_{\mathbf{j}_{\mathbf{k}}}\right|
\end{gathered}
$$

Lemma 7. For any $t=1, \ldots, k$, if $\left|\left\langle\mathbf{a}_{\mathbf{j}_{\mathbf{t}}}, \xi\right\rangle\right|<1$ then in notation (29)-(34)

$$
\frac{1}{\prod_{t=1}^{k}\left(1-\left\langle\mathbf{a}_{\mathbf{j}_{\mathbf{t}}}, \xi\right\rangle\right)^{i_{t}+1}}=\frac{1}{I_{k} !} \sum_{\mu \geqslant 0}\left(\sum_{\mathbf{m}_{\mathbf{J}_{\mathbf{k}}}=\mu} \frac{\prod_{t=1}^{k}\left(\left|\mathbf{m}_{\mathbf{j}_{\mathbf{t}}}\right|+i_{t}\right) !}{\mathbf{m}_{\mathbf{J}_{\mathbf{k}}} !} \mathbf{a}_{\mathbf{J}_{\mathbf{k}}} \mathbf{m}_{\mathbf{J}_{\mathbf{k}}}\right) \xi^{\mu},
$$

where $\mu=\left(\mu_{1}, \ldots, \mu_{n}\right) \in \mathbb{Z}_{+}^{n}$.

Proof of lemma \%. Recall that in case $|\langle a, \xi\rangle|<1$ the following formula holds

$$
\begin{aligned}
& \frac{1}{(1-\langle a, \xi\rangle)^{i}=} 1+\frac{(-i)(-\langle a \xi\rangle)^{1}}{1 !}+\frac{(-i)(-i-1)(-\langle a \xi\rangle)^{2}}{2 !}+ \\
& \ldots+\frac{(-i)(-i-1) \ldots(-i-(l-1))(-\langle a \xi\rangle)^{l}}{l !}+\ldots= \\
&= \sum_{l=0}^{\infty} \frac{(l+i-1) !}{l !(i-1) !}(\langle a \xi\rangle)^{l}=\sum_{l=0}^{\infty} \frac{(l+i-1) !}{l !(i-1) !}\left(a_{1} \xi_{1}+\ldots+a_{n} \xi_{n}\right)^{l}= \\
&= \sum_{m=0}^{\infty} \frac{(l+i-1) !}{l !(i-1) !} \sum_{m_{1}+\ldots+m_{n}=l} \frac{m !}{m_{1} ! \ldots m_{n} !}\left(a_{1} \xi_{1}\right)^{m_{1}} \cdot \ldots \cdot\left(a_{n} \xi_{n}\right)^{m_{n}}= \\
&=\sum_{|m|=0}^{\infty} \frac{(|m|+i-1) !}{m !(i-1) !}(a \xi)^{m}
\end{aligned}
$$

where $m=\left(m_{1}, \ldots, m_{n}\right), \quad(a \xi)^{m}=\left(a_{1} \xi_{1}\right)^{m_{1}} \cdot \ldots \cdot\left(a_{n} \xi_{n}\right)^{m_{n}}=a^{m} \xi^{m}$ and $|m|=m_{1}+\ldots+m_{n}$.

Let $\left|\left\langle\mathbf{a}_{\mathbf{j}_{\mathbf{t}}}, \xi\right\rangle\right|<1, t=1, \ldots, k$. Applying (36), we get

$$
\begin{aligned}
& \prod_{t=1}^{k} \frac{1}{\left(1-\left\langle\mathbf{a}_{\mathbf{j}_{\mathbf{t}}}, \xi\right\rangle\right)^{i_{t}+1}}=\prod_{t=1}^{k}\left(\frac{1}{i_{t} !} \sum_{\left|\mathbf{m}_{\mathbf{j}_{\mathbf{t}}}\right|=0}^{\infty} \frac{\left(\left|\mathbf{m}_{\mathbf{j}_{\mathbf{t}}}\right|+i_{t}\right) !}{\mathbf{m}_{\mathbf{j}_{\mathbf{t}}} !}\left(\mathbf{a}_{\mathbf{j}_{\mathbf{t}}} \xi\right)^{\mathbf{m}_{\mathbf{j}_{\mathbf{t}}}}\right)= \\
& =\frac{1}{I_{k} !} \sum_{\left|\mathbf{m}_{\mathbf{j}_{\mathbf{1}}}\right|=0}^{\infty} \cdots \sum_{\left|\mathbf{m}_{\mathbf{j}_{\mathbf{k}}}\right|=0}^{\infty}\left(\prod_{t=1}^{k} \frac{\left(\left|\mathbf{m}_{\mathbf{j}_{\mathbf{t}}}\right|+i_{t}\right) !}{\mathbf{m}_{\mathbf{j}_{\mathbf{t}}} !}\left(\mathbf{a}_{\mathbf{j}_{\mathbf{t}}} \xi\right)^{\mathbf{m}_{\mathbf{j}_{\mathbf{t}}}}\right)= \\
& =\frac{1}{I_{k} !} \sum_{\left|\mathbf{m}_{\mathbf{j}_{\mathbf{1}}}\right|=0}^{\infty} \ldots \sum_{\left|\mathbf{m}_{\mathbf{j}_{\mathbf{k}}}\right|=0}^{\infty}\left(\prod_{t=1}^{k} \frac{\left(\left|\mathbf{m}_{\mathbf{j}_{\mathbf{t}}}\right|+i_{t}\right) !}{\mathbf{m}_{\mathbf{j}_{\mathbf{t}}} !}\left(\mathbf{a}_{\mathbf{j}_{\mathbf{t}}}^{\mathbf{m}_{\mathbf{j}_{\mathbf{t}}}}\right)\right) \xi^{\left(\mathbf{m}_{\mathbf{j}_{\mathbf{1}}}+\ldots+\mathbf{m}_{\mathbf{j}_{\mathbf{k}}}\right)}= \\
& =\frac{1}{I_{k} !} \sum_{\mathbf{m}_{\mathbf{J}_{\mathbf{k}}} \geqslant 0} \frac{\prod_{t=1}^{k}\left(\left|\mathbf{m}_{\mathbf{j}_{\mathbf{t}}}\right|+i_{t}\right) !}{\mathbf{m}_{\mathbf{J}_{\mathbf{k}}} !} \mathbf{a}_{\mathbf{J}_{\mathbf{k}}}^{\mathbf{m}_{\mathbf{J}_{\mathbf{k}}}} \xi^{\mathbf{m}_{\mathbf{J}_{\mathbf{k}}}}=\frac{1}{I_{k} !} \sum_{\mu \geqslant 0}\left(\sum_{\mathbf{m}_{\mathbf{J}_{\mathbf{k}}=\mu}} \frac{\prod_{t=1}^{k}\left(\left|\mathbf{m}_{\mathbf{j}_{\mathbf{t}}}\right|+i_{t}\right) !}{\mathbf{m}_{\mathbf{J}_{\mathbf{k}}} !} \mathbf{a}_{\mathbf{J}_{\mathbf{k}}}\right) \xi^{\mu} \text {. }
\end{aligned}
$$


Proof of theorem 6. Apply the theorem from [1] to the holomorphic function $f(z)=z^{s}=z_{1}^{s_{1}}$. $\ldots \cdot z_{n}^{s_{n}},\left(s_{1}, \ldots, s_{n}\right) \in \mathbb{Z}_{+}^{n}$.

On every edge $S^{J_{k}}$ by virtue of (10) we then obtain

$$
\begin{aligned}
\nu^{j_{1} \ldots j_{k}}=\frac{I_{k} !}{(2 \pi i)^{n}} \int_{\left|S^{J_{k}}\right|} \tilde{L}_{I_{k}}\left(g^{J_{k}}\right) \omega_{J_{k}} \int_{|\xi|=1} \frac{\left(\frac{\left|\zeta_{1}\right|}{\xi_{1}}\right)^{s_{1}} \cdot \ldots \cdot\left(\frac{\left|\zeta_{n}\right|}{\xi_{n}}\right)^{s_{n}}}{\prod_{t=1}^{k}\left\langle\nabla g_{|\zeta|}^{j_{t}},|\zeta|-\xi z\right\rangle^{i_{t}+1}} \cdot \frac{d \xi_{1}}{\xi_{1}} \wedge \ldots \wedge \frac{d \xi_{n}}{\xi_{n}}= \\
=\frac{I_{k} !}{(2 \pi i)^{n}} \int_{\left|S^{J_{k}}\right|} \tilde{L}_{I_{k}}\left(g^{J_{k}}\right) \omega_{J_{k}} \int_{|\xi|=1} \frac{|\zeta|^{s}}{\xi^{s} \prod_{t=1}^{k} A_{j_{t}}^{i_{t}+1}\left(1-\left\langle\mathbf{a}_{\mathbf{j}_{\mathbf{t}}}, \xi\right\rangle\right)^{i_{t}+1}} \cdot \frac{d \xi}{\xi} .
\end{aligned}
$$

Due to completeness of the domain, it follows from Remark 5 and formula (25), for $t=1, \ldots, k$ that $\left|\left\langle\mathbf{a}_{\mathbf{j}_{\mathbf{t}}}, \xi\right\rangle\right|<1$, where $\left|\xi_{1}\right|=1, \ldots,\left|\xi_{n}\right|=1$. Applying (35) we obtain from (37) that

$$
\begin{aligned}
& =\frac{I_{k} !}{(2 \pi i)^{n}} \int_{|\xi|=1} \frac{1}{\xi^{s} \prod_{t=1}^{k} A_{j_{t}}^{i_{t}+1}} \cdot \frac{1}{I_{k} !} \sum_{\mu \geq 0}\left(\sum_{\mathbf{m}_{\mathbf{J}_{\mathbf{k}}}=\mu} \frac{\left(\left|\mathbf{m}_{\mathbf{j}_{\mathbf{1}}}\right|+i_{1}\right) ! \cdot \ldots \cdot\left(\left|\mathbf{m}_{\mathbf{j}_{\mathbf{k}}}\right|+i_{k}\right) !}{\mathbf{m}_{\mathbf{J}_{\mathbf{k}}} !} \mathbf{a}_{\mathbf{J}_{\mathbf{k}}}\right) \xi^{\mu} \cdot \frac{d \xi}{\xi}= \\
& =\frac{1}{\prod_{t=1}^{k} A_{j_{t}}^{i_{t}+1}} \sum_{\mathbf{m}_{\mathbf{J}_{\mathbf{k}}}=s} \frac{\left(\left|\mathbf{m}_{\mathbf{j}_{1}}\right|+i_{1}\right) ! \cdot \ldots \cdot\left(\left|\mathbf{m}_{\mathbf{j}_{\mathbf{k}}}\right|+i_{k}\right) !}{\mathbf{m}_{\mathbf{J}_{\mathbf{k}}} !} \mathbf{a}_{\mathbf{J}_{\mathbf{k}}} \mathbf{m}_{\mathbf{J}_{\mathbf{k}}}= \\
& =\frac{1}{\prod_{t=1}^{k} A_{j_{t}}^{i_{t}+1}} \sum_{\mathbf{m}_{\mathbf{J}_{\mathbf{k}}}=s} \frac{\left(\left|\mathbf{m}_{\mathbf{j}_{\mathbf{1}}}\right|+i_{1}\right) ! \cdot \ldots \cdot\left(\left|\mathbf{m}_{\mathbf{j}_{\mathbf{k}}}\right|+i_{k}\right) !}{\mathbf{m}_{\mathbf{J}_{\mathbf{k}}} !} \prod_{\tau=1}^{k} \mathbf{a}_{\mathbf{j}_{\tau}}^{\mathbf{m}_{\mathbf{j}_{\tau}}}= \\
& =\frac{1}{\prod_{t=1}^{k} A_{j_{t}}^{i_{t}+1}} \sum_{\mathbf{m}_{\mathbf{J}_{\mathbf{k}}}=s} \frac{\left(\left|\mathbf{m}_{\mathbf{j}_{1}}\right|+i_{1}\right) ! \cdot \ldots \cdot\left(\left|\mathbf{m}_{\mathbf{j}_{\mathbf{k}}}\right|+i_{k}\right) !}{\mathbf{m}_{\mathbf{J}_{\mathbf{k}}} !} \prod_{\tau=1}^{k}\left(\frac{g_{|\zeta|}^{j_{\tau}} z}{A_{j_{\tau}}}\right)^{\mathbf{m}_{\mathbf{j}_{\tau}}}= \\
& =\frac{z^{s}}{\prod_{t=1}^{k} A_{j_{t}}^{\left|\mathbf{m}_{\mathbf{j}_{\mathbf{t}}}\right|+i_{t}+1}} \sum_{\mathbf{m}_{\mathbf{J}_{\mathbf{k}}=s}} \frac{\left(\left|\mathbf{m}_{\mathbf{j}_{\mathbf{1}}}\right|+i_{1}\right) ! \cdot \ldots \cdot\left(\left|\mathbf{m}_{\mathbf{j}_{\mathbf{k}}}\right|+i_{k}\right) !}{\mathbf{m}_{\mathbf{J}_{\mathbf{k}}} !} \prod_{\tau=1}^{k}\left(g_{|\zeta|}^{j_{\tau} \mid}\right)^{\mathbf{m}_{\mathbf{j}_{\tau}}} .
\end{aligned}
$$

Then (37) becomes

$$
\nu^{j_{1} \ldots j_{k}}=z^{s} \cdot \sum_{\mathbf{m}_{\mathbf{J}_{\mathbf{k}}}=s} \frac{\left(\left|\mathbf{m}_{\mathbf{j}_{\mathbf{1}}}\right|+i_{1}\right) ! \cdot \ldots \cdot\left(\left|\mathbf{m}_{\mathbf{j}_{\mathbf{k}}}\right|+i_{k}\right) !}{\mathbf{m}_{\mathbf{J}_{\mathbf{k}}} !} \int_{\left|S^{J_{k}}\right|} \frac{|\zeta|^{s} \tilde{L}_{I_{k}}\left(g^{J_{k}}\right)}{\prod_{t=1}^{k} A_{j_{t}}^{\left|\mathbf{m}_{\mathbf{m}_{\mathbf{t}}}\right|+i_{t}+1}} \prod_{\tau=1}^{k}\left(g_{|\zeta|}^{j_{\tau}}\right)^{\mathbf{m}_{\mathbf{j}_{\tau}}} \omega_{J_{k}},
$$

or

$$
\nu^{j_{1} \ldots j_{k}}=z^{s} \cdot \sum_{\mathbf{m}_{\mathbf{J}_{\mathbf{k}}}=s} \frac{\left(\left|\mathbf{m}_{\mathbf{j}_{1}}\right|+i_{1}\right) ! \cdot \ldots \cdot\left(\left|\mathbf{m}_{\mathbf{j}_{\mathbf{k}}}\right|+i_{k}\right) !}{\mathbf{m}_{\mathbf{J}_{\mathbf{k}}} !} \cdot \Omega_{\mathbf{m}_{\mathbf{J}_{\mathbf{k}}}}\left(s, J_{k}, I_{k}\right)
$$

where

$$
\begin{gathered}
\Omega_{\mathbf{m}_{\mathbf{J}_{\mathbf{k}}}}\left(s, J_{k}, I_{k}\right)=\int_{\mid S^{J_{k} \mid}} \frac{|\zeta|^{s} \tilde{L}_{I_{k}}\left(g^{J_{k}}\right)}{\prod_{t=1}^{k} A_{j_{t}}^{\left|\mathbf{m}_{\mathbf{j}_{\mathbf{t}}}\right|+i_{t}+1}} \prod_{\tau=1}^{k}\left(g_{|\zeta|}^{j_{\tau}}\right)^{\mathbf{m}_{\mathbf{j}_{\tau}}} \omega_{J_{k}}= \\
=\int_{\mid S^{J_{k} \mid}} \frac{|\zeta|^{s}\left(g_{|\zeta|}^{j_{1}}\right)^{\mathbf{m}_{\mathbf{j}_{1}}} \cdot \ldots \cdot\left(g_{|\zeta|}^{j_{k}}\right)^{\mathbf{m}_{\mathbf{j}_{\mathbf{k}}}}}{\prod_{t=1}^{k} A_{j_{t}}^{\left|\mathbf{m}_{\mathbf{j}_{\mathbf{t}}}\right|+i_{t}+1}} \cdot \tilde{L}_{I_{k}}\left(g^{J_{k}}\right) \omega_{J_{k}} .
\end{gathered}
$$


Applying (39) to (9) of theorem [1], for $f(z)=z^{s}$, we obtain

$$
z^{s}=z^{s}(-1)^{n} \sum_{k=1}^{n}(-1)^{k-1} \sum_{\sharp J_{k}=k}{ }^{\prime} \sum_{\left|I_{k}\right|=n-k} \sum_{\mathbf{m}_{\mathbf{J}_{\mathbf{k}}}=s} \frac{\left(\left|\mathbf{m}_{\mathbf{j}_{1}}\right|+i_{1}\right) ! \cdot \ldots \cdot\left(\left|\mathbf{m}_{\mathbf{j}_{\mathbf{k}}}\right|+i_{k}\right) !}{\mathbf{m}_{\mathbf{J}_{\mathbf{k}}} !} \Omega_{\mathbf{m}_{\mathbf{J}_{\mathbf{k}}}}\left(s, J_{k}, I_{k}\right) .
$$

Whence a series of identities follows

$$
1 \equiv(-1)^{n} \sum_{k=1}^{n}(-1)^{k-1} \sum_{\sharp J_{k}=k} \sum_{\left|I_{k}\right|=n-k} \sum_{\mathbf{m}_{\mathbf{J}_{\mathbf{k}}}=s} \frac{\left(\left|\mathbf{m}_{\mathbf{j}_{1}}\right|+i_{1}\right) ! \cdot \ldots \cdot\left(\left|\mathbf{m}_{\mathbf{j}_{\mathbf{k}}}\right|+i_{k}\right) !}{\mathbf{m}_{\mathbf{J}_{\mathbf{k}}} !} \Omega_{\mathbf{m}_{\mathbf{J}_{\mathbf{k}}}}\left(s, J_{k}, I_{k}\right) .
$$

I would like to express my deep apprecation to Dr. E. Leinartas, Dr. G. Egorychev and Dr. A. Shchuplev for their helpful advices and remarks.

I am also grateful to Dr. S. LJ. Damjanovic for the works $[6,9,10]$ which he referred me to.

The work was supported by the grant of the Russian Federation Goverment for research under the supervision of leading scientist at the Siberian Federal Univesity, contract 14.Y26.31.0006.

\section{References}

[1] V.P.Krivokolesko, Integral representations for linearly convex polyhedra and some combinatorial identities, J. Sib. Fed. Univ. Math. Phys., 2(2009), no. 2, 176-188 (in Russian).

[2] T.W.Chaundy, J.E Bullard, John Smith's problem, Math. Gazette, 44(1960), 253-260.

[3] V.M.Shelkovich, Algebra of distributions with a point singular support, Dokl. AN SSSR, 267(1)(1982), 53-57 (in Russian).

[4] I.Daubeacheis, Ten Lectures on Wavelets, SIAM, Philadelphia, 1992.

[5] D.Zielberger, On an identity of Daubeachies, Amer. Math. Monthly, 100(1993), 487.

[6] T.H.Koornwinder, M.J. Schlosser, On an identity Chaundy and Bullard. I, Indag. Math. (N.S), 19(2008), 239-261.

[7] G.P.Egorychev, Combinatorial identity from the theory of integral representations in $\mathbb{C}^{n}$, Irkutsk Gos. Univ. Mat., 4(4)(2011), 32-44 (in Russian).

[8] V.P.Krivokolesko, E.K.Leinartas, On identities with polynomial coefficients, Irkutsk Gos. Univ. Mat., 5(3)(2012), 56-63 (in Russian).

[9] T.H.Koornwinder, M.J.Schlosser, On an identity Chaundy and Bullard. II, More history, Indag. Math. (N.S), 24(2013), 174-180.

[10] H.Alzer, On a combiatorial sum, Indag. Math., 26(2015), 519-525.

[11] V.P.Krivokolesko, Integral representation of holomophic functions in n-circular linear convex domain with piecewise regular boudary, Mat. sistemy, Krasnoyarsk, Izd. KrasGAU, 10(2011), 95-107 (in Russian).

[12] G.P.Egorychev, Integral Representation and the Computation of Combinatorial Sums, Trans. of Math. Monogr., vol. 59, Amer. Math. Soc., Providence, RI, 1984. 
[13] M.N.Davletshin, G.P.Egorychev, V.P.Krivokolesko, Calculation of multiple combinatorial sums in the theory of holomorphic functions in $\mathbb{C}^{n}$, Advances in Applied Mathematics, 48(2012), 446-456.

[14] V.P.Krivokolesko, A.K.Tsikh, Integral representations in linearly convex polyhedra, Sib. Mat. J., 46(2005), no. 3, 579-593.

[15] L.A.Aizenberg, A.P.Yuzhakov, Integral Represetations and Residues in Multidimensional Complex Analysis, AMS, RI, Providence, 1983.

[16] M.Andersson, M.Passare, R.Sigurdsson, Complex convexity and analytic functionals, BaselBoston-Berlin, Birkhäuser, (Progr. Math., 225), 2004.

[17] M.Forsberg, M.Passare, A.Tsikh, Laurent Determinants and Arrangements of Hyperplane Amoebas, Advances in Mathematics, 151(2000), 45-70.

\title{
Получение комбинаторных тождеств с полиномиальными коэффициентами с помощью интегрального представле- ния
}

\author{
Вячеслав П. Кривоколеско \\ Институт математики и фундаментальной информатики \\ Сибирский федеральный университет \\ Свободный, 79, Красноярск, 660041 \\ Россия
}

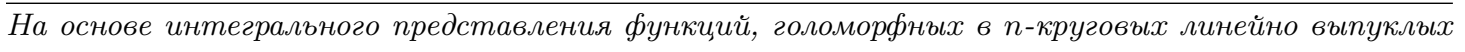
полиэдрах, предложен метод получения тождеств с полиномиальными коэфбициентами.

Ключевые слова: комбинаторные тождества, полиномиальные коэффициенты, интегральные представления. 